Research Article

\title{
Logarithmical Regularity Criteria of the Three-Dimensional Micropolar Fluid Equations in terms of the Pressure
}

\author{
Yan Jia, Jing Zhang, and Bo-Qing Dong \\ School of Mathematical Sciences, Anhui University, Hefei 230601, China \\ Correspondence should be addressed to Bo-Qing Dong, bqdong@ahu.edu.cn
}

Received 21 May 2012; Accepted 19 June 2012

Academic Editor: Yonghong Yao

Copyright (C) 2012 Yan Jia et al. This is an open access article distributed under the Creative Commons Attribution License, which permits unrestricted use, distribution, and reproduction in any medium, provided the original work is properly cited.

This paper is devoted to the regularity criterion of the three-dimensional micropolar fluid equations. Some new regularity criteria in terms of the partial derivative of the pressure in the Lebesgue spaces and the Besov spaces are obtained which improve the previous results on the micropolar fluid equations.

\section{Introduction and Main Result}

In this paper, we consider a three-dimensional micropolar fluid equations model described by the following equations:

$$
\begin{gathered}
\nabla \cdot u=0, \\
\partial_{t} u-\Delta u-\nabla \times w+\nabla \pi+u \cdot \nabla u=0, \\
\partial_{t} w-\Delta w-\nabla \nabla \cdot w+2 w-\nabla \times u+u \cdot \nabla w=0,
\end{gathered}
$$

associated with the initial condition

$$
u(x, 0)=u_{0}, \quad w(x, 0)=w_{0},
$$

where $u(x, t)=\left(u_{1}(x, t), u_{2}(x, t), u_{3}(x, t)\right)$ is the unknown velocity vector field, $\pi(x, t)$ is the unknown scalar pressure field, and $w(x, t)=\left(w_{1}(x, t), w_{2}(x, t), w_{3}(x, t)\right)$ is the unknown 
microrotation vector field with $\nabla \cdot u_{0}=0$ in the sense of distribution. $u_{0}$ and $w_{0}$ represent the prescribed initial data for the velocity and microrotation fields.

Here,

$$
\begin{gathered}
\nabla=\left(\partial_{x_{1}}, \partial_{x_{2}}, \partial_{x_{3}}\right), \quad \Delta=\nabla \cdot \nabla \\
\nabla \cdot u=\sum_{i=1}^{3} \partial_{i} u_{i}, \quad u \cdot \nabla w=\sum_{i=1}^{3} u_{i} \partial_{i} w_{j}, \quad(j=1,2,3) .
\end{gathered}
$$

Micropolar fluid equations introduced by Eringen [1] in 1960' are a special model of the non-Newtonian fluids which is coupled with the viscous incompressible Navier-Stokes model, microrotational effects and microrotational inertia. Physically, micropolar fluid may represent fluids that consist of rigid, randomly oriented (or spherical particles), suspended in a viscous medium, where the deformation of fluid particles is ignored. It can describe many phenomena appeared in a large number of complex fluids such as the suspensions, animal blood, and liquid crystals which cannot be characterized appropriately by the NavierStokes system [2]. When the microrotation effects are neglected or $w=0$, the micropolar fluid equations reduces to the classic Navier-Stokes flows (see [3]).

Due to the importance of both physics and mathematics, the questions on the well posedness and asymptotic behaviors of solutions for micropolar fluid equations have been attracted more and more attention. Galdi and Rionero [4], Łukaszewicz [5] considered the existence of weak solutions of the micropolar fluid flows (1.1)-(1.2). the existence of strong solutions with either local for large data or global for small data is considered by many authors [6-9]. One may also refer to the interesting results on the large time behaviors of micropolar fluid equations in [10-13].

However, similar to the classic Navier-Stokes equations, the question of global regularity of the weak solutions of the 3D micropolar fluid equations still remain a big open problem. It is of importance on the study of regularity of the weak solutions under additional critical growth conditions on the velocity or the pressure. Based on some analysis technique, some regularity criteria via the velocity of weak solutions in the Lebesgue spaces, multiplier spaces and Besov spaces have been obtained in [14-16]. In particular, as for the pressure criterion, Dong et al. [17] (see also Yuan [18]) showed that the weak solution becomes regular if the pressure satisfies

$$
\pi \in L^{p}\left(0, T ; L^{q, \infty}\left(\mathbb{R}^{3}\right)\right), \quad \text { for } \frac{2}{p}+\frac{3}{q} \leq 2, \frac{3}{2}<q \leq \infty,
$$

or

$$
\pi \in L^{1}\left(0, T ; B_{\infty, \infty}^{0}\left(\mathbb{R}^{3}\right)\right),
$$

where $L^{q, \infty}$ and $B_{\infty, \infty}^{0}$ denote the Lorentz space and Besov space. Later on, those results of pressure regularity criteria were further improved to the largest critical Besov spaces by Jia et al. [19] as

$$
\pi(x, t) \in L^{p}\left(0, T ; B_{q, \infty}^{r}\left(\mathbb{R}^{3}\right)\right),
$$


where

$$
\frac{2}{p}+\frac{3}{q}=2+r, \quad \frac{3}{2+r}<q<\infty,-1<r \leq 1
$$

One may also refer to some interesting results on the pressure criteria for NavierStokes equations [20-23] and MHD equations [24, 25].

The aim of this paper is to study the logarithmical regularity criterion for the threedimensional micropolar fluid equations in terms of the partial derivative of pressure in the Lebesgue space or pressure in Besov spaces. Before giving the main results, we first recall the definition of weak solutions for micropolar fluid equations (1.1)-(1.2).

Definition 1.1 (Lukaszewicz [5]). Let $\left(u_{0}, w_{0}\right) \in L^{2}\left(\mathbb{R}^{3}\right)$ and $\nabla \cdot u_{0}=0$ in the sense of distribution. A pair vector field $(u(x, t), w(x, t))$ is termed as a weak solution of (1.1)-(1.2) on $(0, T)$, if $(u, w)$ satisfies the following properties:

(i) $(u, w) \in L^{\infty}\left(0, T ; L^{2}\left(\mathbb{R}^{3}\right)\right) \cap L^{2}\left(0, T ; H^{1}\left(\mathbb{R}^{3}\right)\right)$;

(ii) $\nabla \cdot u=0$ in the sense of distribution;

(iii) $(u, w)$ verifies $(1.1)$ in the sense of distribution.

Now our result reads as follows.

Theorem 1.2. Suppose $\left(u_{0}, w_{0}\right) \in L^{4}\left(\mathbb{R}^{3}\right) \cap L^{2}\left(\mathbb{R}^{3}\right)$ and $(u, w)$ is a weak solution of the $3 D$ micropolar fluid equations (1.1)-(1.2). If the partial derivative of the pressure, for example, $\partial_{3} \pi$ satisfies the logarithmical Serrin's type condition

$$
\int_{0}^{T} \frac{\left\|\partial_{3} \pi\right\|_{L^{q}}^{p}}{1+\ln \left(e+\|w\|_{L^{4}}\right)} d s<\infty, \quad \frac{2}{p}+\frac{3}{q}=\frac{7}{4}, \frac{12}{7}<q \leq \infty,
$$

then the weak solution $(u, w)$ becomes a regular solution on $(0, T]$.

Theorem 1.3. On substitution of logarithmical Serrin's type condition (1.8) by the following growth condition

$$
\int_{0}^{T} \frac{\|\pi\|_{\dot{B}_{\infty, \infty}^{-1}}^{2}}{1+\ln \left(e+\|w\|_{L^{4}}\right)} d s<\infty
$$

the conclusion of Theorem 1.2 holds true.

Remark 1.4. It is easy to see that (1.8) on the partial derivative of pressure does not seem comparable with (1.4)-(1.6) on the total pressure at least there is no inclusion relation between them. Moreover, (1.9) can be viewed as an extension of (1.4)-(1.6). Since the growth condition (1.8) is about $\partial_{3} \pi$ and (1.9) is on the Besov space $\dot{B}_{\infty, \infty}^{-1}$, the methods in previous results in [17-19] are not available any more. In order to come over the additional difficulty, the rigorous analysis due to the new structure in nonlinear terms of the system (1.1)-(1.2) is made and some anisotropic function inequalities are also employed. 


\section{Preliminaries}

Throughout this paper, we denote by $C$ the constant which may change from line to line. We also use the following usual function spaces $L^{p}\left(\mathbb{R}^{3}\right), 1 \leq p \leq \infty$, denote the Lebesgue space of all $L^{p}$ integral functions associated with the norm

$$
\|f\|_{L^{p}}= \begin{cases}\left(\int_{\mathbb{R}^{3}}|f(x)|^{p} d x\right)^{1 / p}, & 1 \leq p<\infty, \\ \operatorname{ess} \sup _{x \in \mathbb{R}^{3}}|f(x)|, & p=\infty .\end{cases}
$$

$H^{k}\left(\mathbb{R}^{3}\right)$ denotes the Hilbert space $\left\{u \in L^{2}\left(\mathbb{R}^{3}\right) ;\left\|\nabla^{k} u\right\|_{L^{2}}<\infty\right\}$.

$\dot{B}_{p, q}^{s}\left(\mathbb{R}^{3}\right)$ denotes the homogeneous Besov space which is defined by the full-dyadic decomposition such as

$$
\dot{B}_{p, q}^{s}\left(\mathbb{R}^{3}\right)=\left\{f \in \frac{\mathcal{S}^{\prime}\left(\mathbb{R}^{3}\right)}{p\left(\mathbb{R}^{3}\right)}:\|f\|_{\dot{B}_{p, q}^{s}}<\infty\right\}
$$

where

$$
\|f\|_{\dot{B}_{p, q}^{s}}= \begin{cases}\left(\sum_{j=-\infty}^{\infty} 2^{j s q}\left\|\Delta_{j} f\right\|_{L^{p}}^{q}\right)^{1 / q}, & 1 \leq q<\infty, \\ \sup _{j \in Z} 2^{j s}\left\|\Delta_{j} f\right\|_{L^{p}}, & q=\infty,\end{cases}
$$

and $S^{\prime}\left(\mathbb{R}^{3}\right), P\left(\mathbb{R}^{3}\right)$ are the spaces of all tempered distributions on $\mathbb{R}^{3}$ and the set of all scalar polynomials defined on $\mathbb{R}^{3}$, respectively. It is worthy to note that the homogeneous Besov space $\dot{B}_{2,2}^{s}\left(\mathbb{R}^{3}\right)$ is equivalent to the Hilbert space $H^{s}\left(\mathbb{R}^{3}\right)$.

In order to prove the main results, we need to recall some anisotropic inequalities. The following Sobolev inequality is due to Cao and $\mathrm{Wu}[26]$.

Lemma 2.1 (Cao and $\mathrm{Wu}[26]$ ). Let the three constants $1 \leq \mu, \lambda, \gamma<\infty$ satisfy

$$
1+\frac{3}{\gamma}=\frac{1}{\mu}+\frac{2}{\lambda}
$$

Assume $\phi \in H^{1}\left(\mathbb{R}^{3}\right), \partial_{1} \phi, \partial_{2} \phi \in L^{\lambda}\left(\mathbb{R}^{3}\right)$ and $\partial_{3} \phi \in L^{\mu}\left(\mathbb{R}^{3}\right)$, then there exists a constant $C=C(\mu, \lambda)$ such that

$$
\|\phi\|_{L^{r}} \leq C\left\|\partial_{1} \phi\right\|_{L^{\lambda}}^{1 / 3}\left\|\partial_{2} \phi\right\|_{L^{\lambda}}^{1 / 3}\left\|\partial_{3} \phi\right\|_{L^{\mu}}^{1 / 3} .
$$

We also recall an interpolation inequality in Besov space due to Meyer [27].

Lemma 2.2 (Meyer [27]). Let $\alpha>0, \beta>0$ and $1 \leq p<q<\infty$, we have for all $f \in \mathcal{S}\left(\mathbb{R}^{3}\right)$,

$$
\|f\|_{L^{q}} \leq C\|f\|_{\dot{B}_{\infty, \infty}^{-\alpha}}^{1-\theta}\|f\|_{\dot{B}_{p, p}^{\beta}}^{\theta} \quad \text { for } \beta=\alpha\left(\frac{q}{p}-1\right), \quad \theta=\frac{q}{p} .
$$


The following local existence and uniqueness of the strong solution for 3D micropolar fluid equations obtained by Dong et al. [17].

Lemma 2.3 (Dong et al. [17]). Assume $3<p<\infty$ and $\left(u_{0}, w_{0}\right) \in L^{p}\left(\mathbb{R}^{3}\right)$ with $\nabla \cdot u_{0}=0$ in the sense of distributions. Then there exist a constant $T>0$ and $a$ unique strong solution $(u, w)$ of the $3 D$ micropolar fluid equations (1.1)-(1.2) such that

$$
u \in B C\left([0, T) ; L^{p}\left(\mathbb{R}^{3}\right)\right), t^{1 / 2} \nabla u \in B C\left([0, T) ; L^{p}\left(\mathbb{R}^{3}\right)\right) .
$$

\section{Proof of Main Results}

According to the local existence result in Lemma 2.3, it allows us to construct a unique $L^{4}$-strong solution $(u, w)$ of micropolar fluid equations (1.1)-(1.2) under the initial data $\left(u_{0}, w_{0}\right) \in L^{2}\left(\mathbb{R}^{3}\right) \cap L^{4}\left(\mathbb{R}^{3}\right)$. Moreover, the strong solution can be proved to exist on a maximal time interval using the standard local solution extension technique. For the notation simplicity, we may suppose that the maximal time interval is $[0, T)$. Thus, to prove the main results, it suffices to show that

$$
\lim _{t \rightarrow T}\left(\|u(t)\|_{L^{4}}+\|w(t)\|_{L^{4}}\right)<\infty
$$

This boundedness will be obtained by the following steps.

\subsection{Uniform Estimates of Solutions}

In order to prove the main results, we first need some basic estimates of the solutions for the 3D micropolar fluid equations (1.1)-(1.2).

Taking the inner product of the second equation of (1.1) with $u|u|^{2}$ and integrating by parts, it follows that

$$
\frac{1}{4} \frac{d}{d t}\|u\|_{L^{4}}^{4}+\||u| \nabla u\|_{L^{2}}^{2}+\frac{1}{2}\left\|\nabla|u|^{2}\right\|_{L^{2}}^{2}=\int_{\mathbb{R}^{3}}(\nabla \times w) \cdot u|u|^{2} d x-\int_{\mathbb{R}^{3}} u \cdot \nabla \pi|u|^{2} d x
$$

where we have used the following facts:

$$
\begin{gathered}
\int_{\mathbb{R}^{3}}(u \cdot \nabla u) \cdot u|u|^{2} d x=0 \\
(\nabla u) \cdot\left(\nabla\left(u|u|^{2}\right)\right)=|\nabla u|^{2}|u|^{2}+\left.\left.\frac{1}{2}|\nabla| u\right|^{2}\right|^{2} .
\end{gathered}
$$

Similarly, taking the inner product of the second equation of (1.1) with $|w|^{2} w$ and integrating by parts yield

$$
\frac{1}{4} \frac{d}{d t}\|w\|_{L^{4}}^{4}+\||w| \nabla w\|_{L^{2}}^{2}+\frac{1}{2}\||w| \nabla \cdot w\|_{L^{2}}^{2}+\left\|\nabla|w|^{2}\right\|_{L^{2}}^{2}=\int_{\mathbb{R}^{3}}(\nabla \times u) \cdot w|w|^{2} d x-2\|w\|_{L^{4}}^{4}
$$


where we have used

$$
\begin{gathered}
\int_{\mathbb{R}^{3}}(u \cdot \nabla w) \cdot w|w|^{2} d x=0 \\
(\nabla w) \cdot\left(\nabla\left(w|w|^{2}\right)\right)=|\nabla w|^{2}|w|^{2}+\left.\left.\frac{1}{2}|\nabla| w\right|^{2}\right|^{2}, \\
\int_{\mathbb{R}^{3}}(\nabla \cdot w) \nabla \cdot\left(w|w|^{2}\right) d x=\int_{\mathbb{R}^{3}}|\nabla \cdot w|^{2}|w|^{2} d x+\int_{\mathbb{R}^{3}}(\nabla \cdot w) w \cdot \nabla|w|^{2} d x \\
\geq \frac{1}{2} \int_{\mathbb{R}^{3}}|\nabla \cdot w|^{2}|w|^{2} d x-\left.\left.\frac{1}{2} \int_{\mathbb{R}^{3}}|\nabla| w\right|^{2}\right|^{2} d x .
\end{gathered}
$$

Combination of (3.2) and (3.4) derives

$$
\begin{aligned}
& \frac{1}{4} \frac{d}{d t}\left(\|u\|_{L^{4}}^{4}+\|w\|_{L^{4}}^{4}\right)+\||u| \nabla u\|_{L^{2}}^{2}+\frac{1}{2}\left\|\nabla|u|^{2}\right\|_{L^{2}}^{2}+\||w| \nabla w\|_{L^{2}}^{2}+2\|w\|_{L^{4}}^{4} \\
& \quad \leq \int_{\mathbb{R}^{3}}(\nabla \times w) \cdot u|u|^{2} d x+\int_{\mathbb{R}^{3}}(\nabla \times u) \cdot w|w|^{2} d x-\int_{\mathbb{R}^{3}} u \cdot \nabla \pi|u|^{2} d x
\end{aligned}
$$

With the use of Hölder's inequality, Young's inequality, and integration by parts, the first two terms on the right-hand side of (3.6) is bounded by

$$
\begin{aligned}
& \int_{\mathbb{R}^{3}}(\nabla \times w) \cdot u|u|^{2} d x+\int_{\mathbb{R}^{3}}(\nabla \times u) \cdot w|w|^{2} d x \\
& \quad \leq\|w\|_{L^{4}}\||u| \nabla u\|_{L^{2}}\|u\|_{L^{4}}+\|u\|_{L^{4}}\||w| \nabla w\|_{L^{2}}\|w\|_{L^{4}} \\
& \quad \leq C\left(\|u\|_{L^{4}}^{4}+2\|w\|_{L^{4}}^{4}\right)+\frac{1}{2}\left(\||w| \nabla w\|_{L^{2}}^{2}+\||u| \nabla u\|_{L^{2}}^{2}\right) .
\end{aligned}
$$

Inserting (3.7) into (3.6), we obtain

$$
\begin{aligned}
& \frac{d}{d t}\left(\|u\|_{L^{4}}^{4}+\|w\|_{L^{4}}^{4}\right)+2\||u| \nabla u\|_{L^{2}}^{2}+2\left\|\nabla|u|^{2}\right\|_{L^{2}}^{2} \\
& \leq C\left(\|u\|_{L^{4}}^{4}+\|w\|_{L^{4}}^{4}\right)+\left.C\left|\int_{\mathbb{R}^{3}} u \cdot \nabla \pi\right| u\right|^{2} d x \mid \\
& \quad:=C\left(\|u\|_{L^{4}}^{4}+\|w\|_{L^{4}}^{4}\right)+I .
\end{aligned}
$$


In order to estimate $I$, we first establish some estimates between the pressure and the velocity. Taking the operator div on both sides of the second equation of (1.1) gives

$$
\begin{gathered}
\pi=(-\Delta)^{-1} \sum_{i, j=1}^{3} \frac{\partial^{2}}{\partial x_{i} \partial x_{j}}\left(u_{i} u_{j}\right), \\
\nabla \pi=(-\Delta)^{-1} \sum_{i, j=1}^{3} \frac{\partial^{2}}{\partial x_{i} \partial x_{j}}\left(\nabla\left(u_{i} u_{j}\right)\right) .
\end{gathered}
$$

Applying $L^{p}(1<p<\infty)$ boundedness of the singular integral operators yields

$$
\|\pi\|_{L^{p}} \leq C\|u\|_{L^{p}}^{2}, \quad\|\nabla \pi\|_{L^{p}} \leq C\|u \cdot \nabla u\|_{L^{p}} .
$$

Then employing the integration by parts and Hölder's inequality, we estimate $I$ as

$$
\begin{aligned}
I & =\left.C\left|\int_{\mathbb{R}^{3}} u \cdot \nabla \pi\right| u\right|^{2} d x \mid \\
& =C\left|\int_{\mathbb{R}^{3}} \pi\left(u \cdot \nabla|u|^{2}\right) d x\right| \\
& \leq\left. C \int_{\mathbb{R}^{3}}|\pi u||\nabla| u\right|^{2} \mid d x \\
& \leq C\|\pi u\|_{L^{2}}\left\|\nabla|u|^{2}\right\|_{L^{2}} \\
& \leq C\|\pi\|_{L^{4}}\|u\|_{L^{4}}\left\|\nabla|u|^{2}\right\|_{L^{2}} .
\end{aligned}
$$

Now we begin to prove Theorems 1.2 and 1.3, respectively.

\subsection{Proof of Theorem 1.2}

Applying Lemma 2.1 to $\|\pi\|_{L^{4}}$ by choosing $\gamma=4, \mu=q$ such that

$$
\frac{1}{q}+\frac{2}{\lambda}=\frac{7}{4}
$$

Thus, we have

$$
\|\pi\|_{L^{4}} \leq C\left\|\partial_{1} \pi\right\|_{L^{\lambda}}^{1 / 3}\left\|\partial_{2} \pi\right\|_{L^{\lambda}}^{1 / 3}\left\|\partial_{3} \pi\right\|_{L^{q}}^{1 / 3} \leq C\|\nabla \pi\|_{L^{\lambda}}^{2 / 3}\left\|\partial_{3} \pi\right\|_{L^{q}}^{1 / 3} .
$$

Thanks to $12 / 7<q \leq \infty$, then

$$
\frac{8}{7} \leq \lambda<\frac{7}{6}
$$


Using (3.10) together with Hölder's inequality and Gagliardo-Nirenberg inequality derives that

$$
\begin{aligned}
\|\nabla \pi\|_{L^{\lambda}} & \leq C\|u \cdot \nabla u\|_{L^{\lambda}} \leq C\|u\|_{L^{2 \lambda /(2-\lambda)}}\|\nabla u\|_{L^{2}} \\
& =C\left\||u|^{2}\right\|_{L^{\lambda /(2-\lambda)}}^{1 / 2}\|\nabla u\|_{L^{2}} \\
& \leq C\left\||u|^{2}\right\|_{L^{2}}^{(3 / \lambda)-(7 / 4)}\left\|\nabla|u|^{2}\right\|_{L^{2}}^{(9 / 4)-(3 / \lambda)}\|\nabla u\|_{L^{2}} \\
& \leq C\|u\|_{L^{4}}^{(6 / \lambda)-(7 / 2)}\left\|\nabla|u|^{2}\right\|_{L^{2}}^{(9 / 4)-(3 / \lambda)}\|\nabla u\|_{L^{2}} .
\end{aligned}
$$

Plugging (3.13) and (3.15) into (3.11) and employing Young's inequality yield that

$$
\begin{aligned}
I & \leq C\left\|\partial_{3} \pi\right\|_{L^{q}}^{1 / 3}\|\nabla u\|_{L^{2}}^{2 / 3}\|u\|_{L^{4}}^{(4 / \lambda)-(4 / 3)}\left\|\nabla|u|^{2}\right\|_{L^{2}}^{(5 / 2)-(2 / \lambda)} \\
& \leq \frac{1}{2}\left\|\nabla|u|^{2}\right\|_{L^{2}}^{2}+C\left\|\partial_{3} \pi\right\|_{L^{q}}^{4 \lambda / 3(4-\lambda)}\|\nabla u\|_{L^{2}}^{8 \lambda /(12-7 \lambda)}\|u\|_{L^{4}}^{16(3-\lambda) / 3(4-\lambda)} \\
& \leq \frac{1}{2}\left\|\nabla|u|^{2}\right\|_{L^{2}}^{2}+C\left(\left\|\partial_{3} \pi\right\|_{L^{q}}^{4 \lambda /(12-7 \lambda)}+\|\nabla u\|_{L^{2}}^{2}\right)\|u\|_{L^{4}}^{16(3-\lambda) / 3(4-\lambda)} \\
& \leq \frac{1}{2}\left\|\nabla|u|^{2}\right\|_{L^{2}}^{2}+C\left(\left\|\partial_{3} \pi\right\|_{L^{q}}^{p}+\|\nabla u\|_{L^{2}}^{2}\right)\left(\|u\|_{L^{4}}^{4}+1\right)
\end{aligned}
$$

where we have used the following facts in the last line:

$$
\frac{16(3-\lambda)}{3(4-\lambda)}<4, \quad \frac{2}{p}+\frac{3}{q}=\frac{7}{4}, \quad \text { that is } p=\frac{4 \lambda}{12-7 \lambda}
$$

Then inserting (3.16) into (3.8) obtains

$$
\begin{aligned}
& \frac{d}{d t}\left(\|u\|_{L^{4}}^{4}+\|w\|_{L^{4}}^{4}\right)+\left\|\nabla|u|^{2}\right\|_{L^{2}}^{2} \\
& \quad \leq C\left(1+\left\|\partial_{3} \pi\right\|_{L^{q}}^{p}+\|\nabla u\|_{L^{2}}^{2}\right)\left(\|u\|_{L^{4}}^{4}+1\right)+C\left(\|u\|_{L^{4}}^{4}+\|w\|_{L^{4}}^{4}\right)
\end{aligned}
$$

or

$$
\begin{aligned}
& \frac{d}{d t}\left(e+\|u\|_{L^{4}}^{4}+\|w\|_{L^{4}}^{4}\right)+\left\|\nabla|u|^{2}\right\|_{L^{2}}^{2} \\
& \quad \leq C\left(1+\left\|\partial_{3} \pi\right\|_{L^{q}}^{p}+\|\nabla u\|_{L^{2}}^{2}\right)\left(e+\|u\|_{L^{4}}^{4}+\|w\|_{L^{4}}^{4}\right) \\
& \quad \leq C\left\|\partial_{3} \pi\right\|_{L^{q}}^{p}\left(\|u\|_{L^{4}}^{4}+\|w\|_{L^{4}}^{4}\right)+C\left(1+\|\nabla u\|_{L^{2}}^{2}\right)\left(e+\|u\|_{L^{4}}^{4}+\|w\|_{L^{4}}^{4}\right) .
\end{aligned}
$$


Denote

$$
J=\left\|\partial_{3} \pi\right\|_{L^{q}}^{p}\left(e+\|u\|_{L^{4}}^{4}+\|w\|_{L^{4}}^{4}\right)
$$

and thanks to

$$
\begin{aligned}
1+\ln \left(e+\|w\|_{L^{4}}\right) & \leq 1+\ln \left(e+\frac{1}{4}\|w\|_{L^{4}}^{4}+\frac{4}{3}\right) \\
& \leq 1+\ln \frac{1}{2}\left(e+\|w\|_{L^{4}}^{4}\right) \leq 1+\ln \frac{1}{2}+\ln \left(e+\|w\|_{L^{4}}^{4}\right) \\
& \leq 1+\ln \left(e+\|w\|_{L^{4}}^{4}\right),
\end{aligned}
$$

we have for $J$

$$
\begin{aligned}
J & =\frac{\left\|\partial_{3} \pi\right\|_{L^{q}}^{p}}{1+\ln \left(e+\|w\|_{L^{4}}\right)}\left(e+\|u\|_{L^{4}}^{4}+X\|u\|_{L^{4}}^{4}\right)\left[1+\ln \left(e+\|w\|_{L^{4}}\right)\right] \\
& \leq \frac{\left\|\partial_{3} \pi\right\|_{L^{q}}^{p}}{1+\ln \left(e+\|w\|_{L^{4}}\right)}\left(e+\|u\|_{L^{4}}^{4}+\|w\|_{L^{4}}^{4}\right)\left[1+\ln \left(e+\|w\|_{L^{4}}^{4}\right)\right] \\
& \leq \frac{\left\|\partial_{3} \pi\right\|_{L^{q}}^{p}}{1+\ln \left(e+\|w\|_{L^{4}}\right)}\left(e+\|u\|_{L^{4}}^{4}+\|w\|_{L^{4}}^{4}\right)\left[1+\ln \left(e+\|u\|_{L^{4}}^{4}+\|w\|_{L^{4}}^{4}\right)\right] .
\end{aligned}
$$

Thus, plugging (3.22) into (3.19) and denoting

$$
\mathcal{K}=e+\|u\|_{L^{4}}^{4}+\|w\|_{L^{4}}^{4}
$$

we have

$$
\frac{d}{d t} \mathcal{K} \leq C \frac{\left\|\partial_{3} \pi\right\|_{L^{q}}^{p}}{1+\ln \left(e+\|w\|_{L^{4}}\right)}\{1+\ln \mathcal{K}\} \mathcal{K}+C\left(1+\|\nabla u\|_{L^{2}}^{2}\right) \mathcal{K} .
$$

Taking Gronwall's inequality into consideration derives

$$
\begin{aligned}
\mathcal{\mathcal { K }} \leq & \ln \left(e+\left\|u_{0}\right\|_{L^{4}}^{4}+\left\|w_{0}\right\|_{L^{4}}^{4}\right) \\
& \times \exp \left\{C \int_{0}^{t} \frac{\left\|\partial_{3} \pi\right\|_{L^{q}}^{p}}{1+\ln \left(e+\|w\|_{L^{4}}\right)}\{1+\ln \mathcal{K}\} d s\right\} \\
& \times \exp \left\{\int_{0}^{t} C\left(1+\|\nabla u\|_{L^{2}}^{2}\right) d s\right\} .
\end{aligned}
$$


Thanks to $(u, w)$ is a weak solution of the 3D micropolar equations (1.1)-(1.2), then

$$
\int_{0}^{T} C\left(1+\|\nabla u\|_{L^{2}}^{2}\right) d s<C T
$$

we rewrite (3.25) as

$$
\begin{aligned}
1+\ln \mathcal{K} \leq C & +\ln \left(e+\left\|u_{0}\right\|_{L^{4}}^{4}+\left\|w_{0}\right\|_{L^{4}}^{4}\right) \\
& +C \int_{0}^{t} \frac{\left\|\partial_{3} \pi\right\|_{L^{q}}^{p}}{1+\ln \left(e+\|w\|_{L^{4}}\right)}\{1+\ln \mathcal{K}\} d s .
\end{aligned}
$$

Applying Gronwall's inequality again yields

$$
\ln \mathcal{K} \leq C\left(u_{0}, w_{0}, T\right) \exp \left\{C \int_{0}^{T} \frac{\left\|\partial_{3} \pi\right\|_{L^{q}}^{p}}{1+\ln \left(e+\|w\|_{L^{4}}\right)} d s\right\},
$$

which implies that

$$
\lim _{t \rightarrow T}\left(\|u(t)\|_{L^{4}}+\|w(t)\|_{L^{4}}\right)<\infty
$$

Hence, we complete the proof of Theorem 1.2.

\subsection{Proof of Theorem 1.3}

Applying Lemma 2.2 to $\|\pi\|_{L^{4}}$ by choosing

$$
q=2, \quad p=4, \quad \alpha=1, \quad \beta=1, \quad \theta=\frac{1}{2}
$$

we have

$$
\begin{aligned}
\|\pi\|_{L_{4}} & \leq C\|\pi\|_{\dot{B}_{\infty, \infty}^{-1}}^{1 / 2}\|\pi\|_{\dot{B}_{2,2}^{1}}^{1 / 2} \\
& \leq C\|\pi\|_{\dot{B}_{\infty, \infty}^{-1}}^{1 / 2}\|\pi\|_{\dot{H}^{1}}^{1 / 2}=C\|\pi\|_{\dot{B}_{\infty, \infty}^{-1}}^{1 / 2}\|\nabla \pi\|_{L^{2}}^{1 / 2} .
\end{aligned}
$$

Plugging the above inequality into (3.11) together with Young's inequality implies

$$
\begin{aligned}
I & \leq C\|\pi\|_{L^{4}}\|u\|_{L^{4}}\left\|\nabla|u|^{2}\right\|_{L^{2}} \\
& \leq C\|\pi\|_{L^{4}}^{2}\|u\|_{L^{4}}^{2}+\frac{1}{2}\left\|\nabla|u|^{2}\right\|_{L^{2}}^{2} \\
& \leq C\|\pi\|_{\dot{B}_{\infty, \infty}^{-1} \infty}\|\nabla \pi\|_{L^{2}}\|u\|_{L^{4}}^{2}+\frac{1}{2}\left\|\nabla|u|^{2}\right\|_{L^{2}}^{2}
\end{aligned}
$$


Abstract and Applied Analysis

$$
\begin{aligned}
& \leq C\|\pi\|_{\dot{B}_{\infty, \infty}^{-1}}\||u| \nabla u\|_{L^{2}}\|u\|_{L^{4}}^{2}+\frac{1}{2}\left\|\nabla|u|^{2}\right\|_{L^{2}}^{2} \\
& \leq C\|\pi\|_{\dot{B}_{\infty, \infty}^{-1}}^{2}\|u\|_{L^{4}}^{4}+\frac{1}{2}\||u| \nabla u\|_{L^{2}}^{2}+\frac{1}{2}\left\|\nabla|u|^{2}\right\|_{L^{2}}^{2} .
\end{aligned}
$$

Then inserting (3.32) into (3.8) derives

$$
\frac{d}{d t}\left(\|u\|_{L^{4}}^{4}+\|w\|_{L^{4}}^{4}\right) \leq C\left(\|u\|_{L^{4}}^{4}+\|w\|_{L^{4}}^{4}\right)+C\|\pi\|_{\dot{B}_{\infty, \infty}^{-1}}^{2}\|u\|_{L^{4}}^{4} .
$$

Thanks to

$$
\begin{aligned}
\|\pi\|_{\dot{B}_{\infty, \infty}^{-1}}^{2}\|u\|_{L^{4}}^{4} & =\frac{C\|\pi\|_{\dot{B}_{\infty, \infty}^{-1}}^{2}}{1+\ln \left(e+\|w\|_{L^{4}}\right)}\|u\|_{L^{4}}^{4}\left[1+\ln \left(e+\|w\|_{L^{4}}\right)\right] \\
& \leq \frac{C\|\pi\|_{\dot{B}_{\infty, \infty}^{-1}}^{2}}{1+\ln \left(e+\|w\|_{L^{2}}\right)}\|u\|_{L^{4}}^{4}\left[1+\ln \left(e+\|w\|_{L^{4}}^{4}\right)\right] \\
& \leq \frac{C\|\pi\|_{\dot{B}_{\infty, \infty}^{-1}}^{2}}{1+\ln \left(e+\|w\|_{L^{2}}\right)}\left(e+\|u\|_{L^{4}}^{4}+\|w\|_{L^{4}}^{4}\right)\left[1+\ln \left(e+\|u\|_{L^{4}}^{4}+\|w\|_{L^{4}}^{4}\right)\right],
\end{aligned}
$$

inserting the above inequality into (3.33) and, similarly, denoting

$$
\mathcal{K}=e+\|u\|_{L^{4}}^{4}+\|w\|_{L^{4}}^{4}
$$

then we have

$$
\frac{d}{d t} \mathcal{K} \leq C \frac{\|\pi\|_{\dot{B}_{\infty, \infty}^{-1}}^{2}}{1+\ln \left(e+\|w\|_{L^{4}}\right)}\{1+\ln \mathcal{K}\} \mathcal{K}
$$

Taking Gronwall, inequality into consideration derives

$$
\mathcal{K} \leq \ln \left(e+\left\|u_{0}\right\|_{L^{4}}^{4}+\left\|w_{0}\right\|_{L^{4}}^{4}\right) \exp \left\{C \int_{0}^{t} \frac{\|\pi\|_{\dot{B}_{\infty, \infty}^{-1}}^{2}}{1+\ln \left(e+\|w\|_{L^{4}}\right)}\{1+\ln \mathcal{K}\} d s\right\}
$$

or

$$
1+\ln \mathcal{K} \leq \ln \left(e+\left\|u_{0}\right\|_{L^{4}}^{4}+\left\|w_{0}\right\|_{L^{4}}^{4}\right)+C \int_{0}^{t} \frac{\|\pi\|_{B_{\infty}^{-1, \infty}}^{2}}{1+\ln \left(e+\|w\|_{L^{4}}\right)}\{1+\ln \mathcal{K}\} d s .
$$


Likewise, applying Gronwall's inequality again implies

$$
\ln \mathcal{K} \leq C\left(u_{0}, w_{0}\right) \exp \left\{C \int_{0}^{T} \frac{\|\pi\|_{B_{\infty, \infty}^{-1}}^{2}}{1+\ln \left(e+\|w\|_{L^{4}}\right)} d s\right\} .
$$

Hence, we derive that

$$
\lim _{t \rightarrow T}\left(\|u(t)\|_{L^{4}}+\|w(t)\|_{L^{4}}\right)<\infty,
$$

which completes the proof of Theorem 1.3.

\section{Acknowledgments}

This work is partially supported by the NSF of China (10801001), NSF of Anhui Province (11040606M02), and is also financed by the 211 Project of Anhui University (KJTD002B, KJJQ005).

\section{References}

[1] A. C. Eringen, "Theory of micropolar fluids," Journal of Mathematics and Mechanics, vol. 16, pp. 1-18, 1966.

[2] S. Popel, A. Regirer, and P. Usick, “A continuum model of blood flow,” Biorheology, vol. 11, pp. 427437, 1974.

[3] R. Temam, Navier-Stokes Equations, Theory and Numerical Analysis, North-Holland, Amsterdam, The Netherlands, 1977.

[4] G. P. Galdi and S. Rionero, "A note on the existence and uniqueness of solutions of the micropolar fluid equations," International Journal of Engineering Science, vol. 15, no. 2, pp. 105-108, 1977.

[5] G. Łukaszewicz, Micropolar Fluids. Theory and Applications, Modeling and Simulation in Science, Engineering and Technology, Birkhäuser, Boston, Mass, USA, 1999.

[6] Q. Chen and C. Miao, "Global well-posedness for the micropolar fluid system in critical Besov spaces," Journal of Differential Equations, vol. 252, no. 3, pp. 2698-2724, 2012.

[7] Z.-M. Chen and W. G. Price, "Decay estimates of linearized micropolar fluid flows in $\mathbb{R}^{3}$ space with applications to $L_{3}$-strong solutions," International Journal of Engineering Science, vol. 44, no. 13-14, pp. 859-873, 2006.

[8] B.-Q. Dong and Z. Zhang, "Global regularity of the 2D micropolar fluid flows with zero angular viscosity," Journal of Differential Equations, vol. 249, no. 1, pp. 200-213, 2010.

[9] M. A. Rojas-Medar, "Magneto-micropolar fluid motion: existence and uniqueness of strong solution," Mathematische Nachrichten, vol. 188, pp. 301-319, 1997.

[10] J. Chen, Z.-M. Chen, and B.-Q. Dong, "Uniform attractors of non-homogeneous micropolar fluid flows in non-smooth domains," Nonlinearity, vol. 20, no. 7, pp. 1619-1635, 2007.

[11] B.-Q. Dong and Z.-M. Chen, "Global attractors of two-dimensional micropolar fluid flows in some unbounded domains," Applied Mathematics and Computation, vol. 182, no. 1, pp. 610-620, 2006.

[12] B.-Q. Dong and Z.-M. Chen, "On upper and lower bounds of higher order derivatives for solutions to the 2D micropolar fluid equations," Journal of Mathematical Analysis and Applications, vol. 334, no. 2, pp. 1386-1399, 2007.

[13] B.-Q. Dong and Z.-M. Chen, "Asymptotic profiles of solutions to the 2D viscous incompressible micropolar fluid flows," Discrete and Continuous Dynamical Systems Series A, vol. 23, no. 3, pp. 765784, 2009.

[14] B.-Q. Dong and Z.-M. Chen, "Regularity criteria of weak solutions to the three-dimensional micropolar flows," Journal of Mathematical Physics, vol. 50, no. 10, Article ID 103525, 13 pages, 2009. 
[15] B.-Q. Dong and W. Zhang, "On the regularity criterion for three-dimensional micropolar fluid flows in Besov spaces," Nonlinear Analysis: Theory, Methods E Applications, vol. 73, no. 7, pp. 2334-2341, 2010.

[16] J. Yuan, "Existence theorem and blow-up criterion of the strong solutions to the magneto-micropolar fluid equations," Mathematical Methods in the Applied Sciences, vol. 31, no. 9, pp. 1113-1130, 2008.

[17] B.-Q. Dong, Y. Jia, and Z.-M. Chen, "Pressure regularity criteria of the three-dimensional micropolar fluid flows," Mathematical Methods in the Applied Sciences, vol. 34, no. 5, pp. 595-606, 2011.

[18] B. Yuan, "On regularity criteria for weak solutions to the micropolar fluid equations in Lorentz space," Proceedings of the American Mathematical Society, vol. 138, no. 6, pp. 2025-2036, 2010.

[19] Y. Jia, W. Zhang, and B.-Q. Dong, "Remarks on the regularity criterion of the 3D micropolar fluid flows in terms of the pressure," Applied Mathematics Letters, vol. 24, no. 2, pp. 199-203, 2011.

[20] L. C. Berselli and G. P. Galdi, "Regularity criteria involving the pressure for the weak solutions to the Navier-Stokes equations," Proceedings of the American Mathematical Society, vol. 130, no. 12, pp. 35853595, 2002.

[21] Q. Chen and Z. Zhang, "Regularity criterion via the pressure on weak solutions to the 3D NavierStokes equations," Proceedings of the American Mathematical Society, vol. 135, no. 6, pp. 1829-1837, 2007.

[22] J. Fan, S. Jiang, and G. Ni, "On regularity criteria for the $n$-dimensional Navier-Stokes equations in terms of the pressure," Journal of Differential Equations, vol. 244, no. 11, pp. 2963-2979, 2008.

[23] Y. Zhou, "On regularity criteria in terms of pressure for the Navier-Stokes equations in $\mathbb{R}^{3}$," Proceedings of the American Mathematical Society, vol. 134, no. 1, pp. 149-156, 2006.

[24] J. Fan, S. Jiang, G. Nakamura, and Y. Zhou, "Logarithmically improved regularity criteria for the Navier-Stokes and MHD equations," Journal of Mathematical Fluid Mechanics, vol. 13, no. 4, pp. 557$571,2011$.

[25] Y. Zhou, "Regularity criteria for the 3D MHD equations in terms of the pressure," International Journal of Non-Linear Mechanics, vol. 41, no. 10, pp. 1174-1180, 2006.

[26] C. Cao and J. Wu, "Two regularity criteria for the 3D MHD equations," Journal of Differential Equations, vol. 248, no. 9, pp. 2263-2274, 2010.

[27] Y. Meyer, "Oscillating patterns in some nonlinear evolution equations," in Mathematical Foundation of Turbulent Viscous Flows, M. Cannone and T. Miyakawa, Eds., vol. 1871 of Lecture Notes in Mathematics, pp. 101-187, Springer, Berlin, Germany, 2006. 


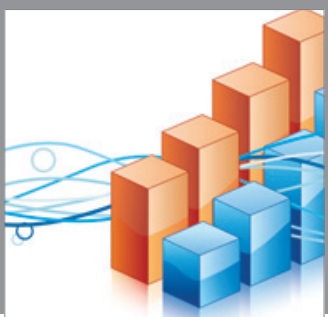

Advances in

Operations Research

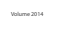

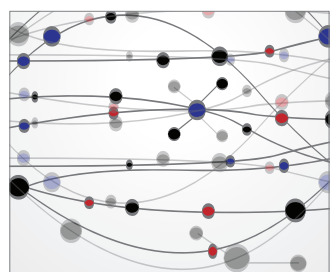

\section{The Scientific} World Journal
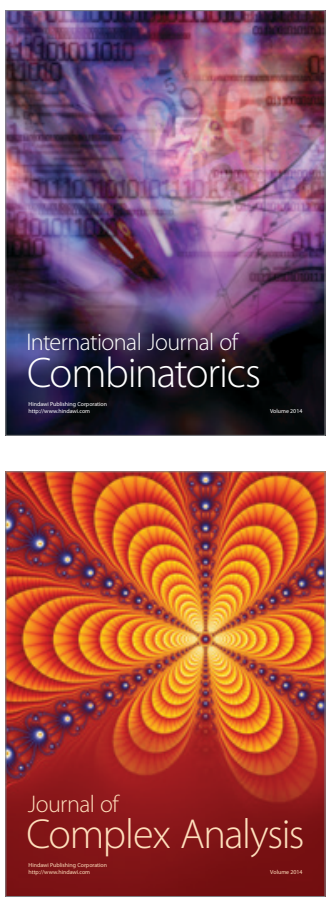

International Journal of

Mathematics and

Mathematical

Sciences
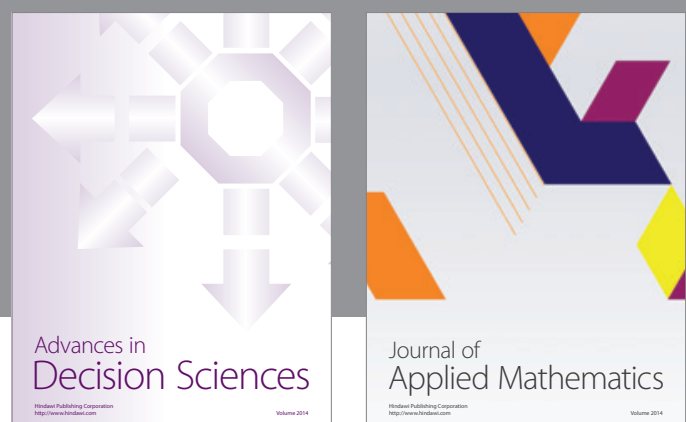

Journal of

Applied Mathematics
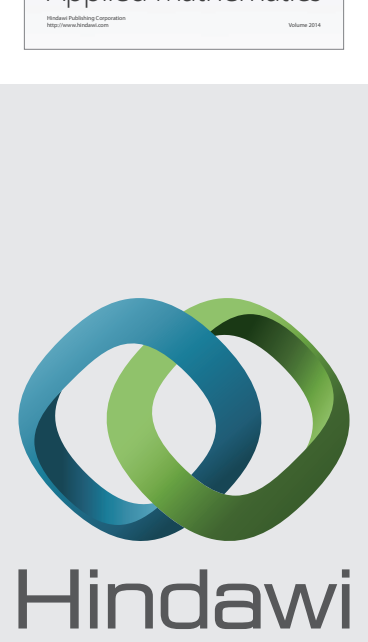

Submit your manuscripts at http://www.hindawi.com
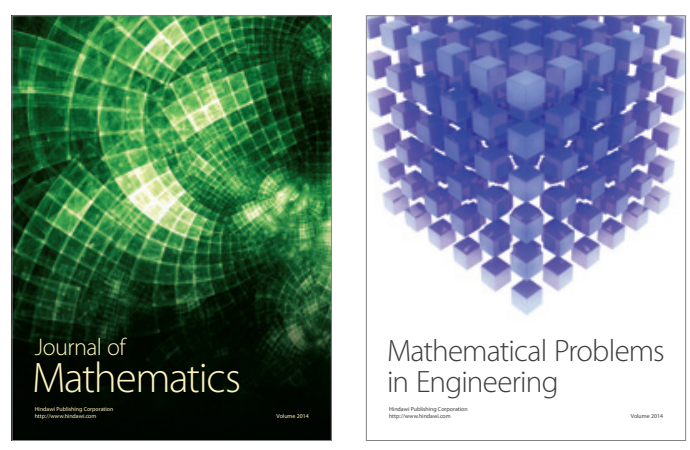

Mathematical Problems in Engineering
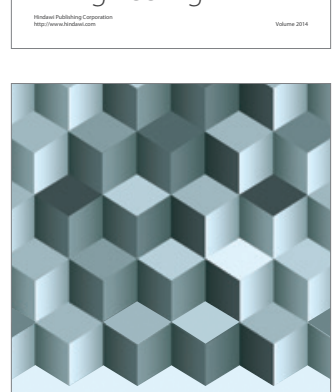

Journal of

Function Spaces
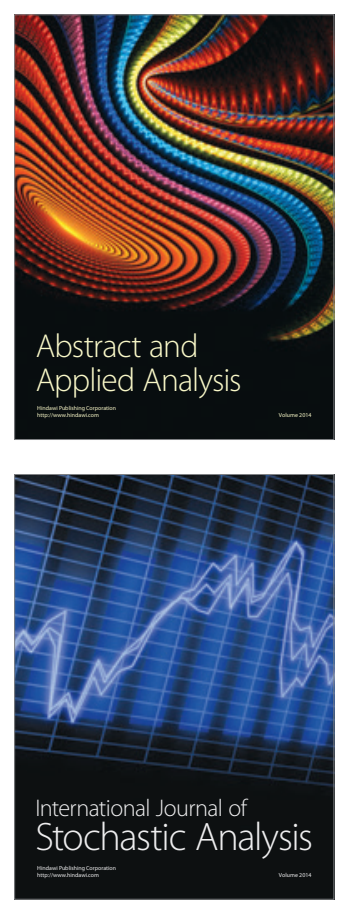

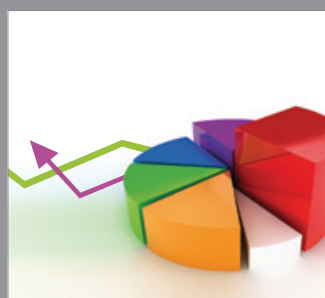

ournal of

Probability and Statistics

Promensencen
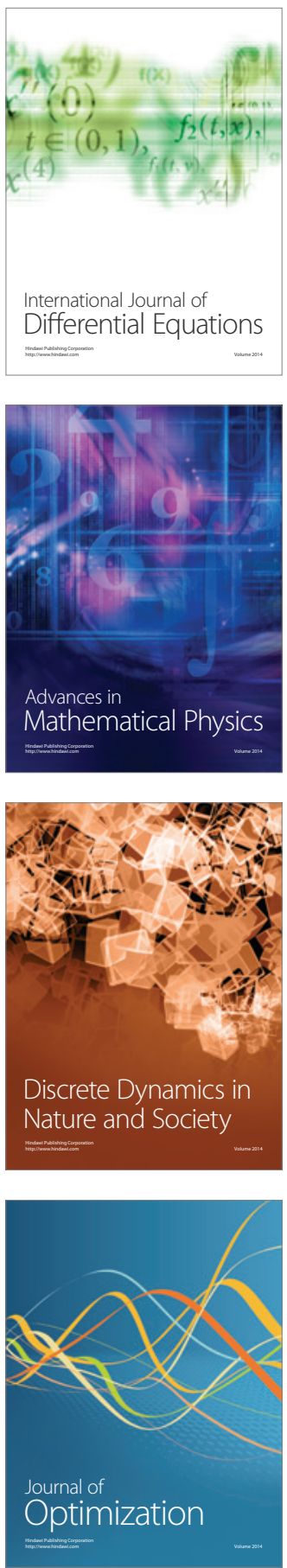